Published in final edited form as:

Urology. 2018 August ; 118: 36-42. doi:10.1016/j.urology.2018.04.036.

\title{
Race- and Sex-related Differences in Nephrolithiasis Risk Among Blacks and Whites in the Southern Community Cohort Study
}

\author{
Ryan S. Hsi, Edmond K. Kabagambe, Xiang Shu, Xijing Han, Nicole L. Miller, Loren \\ Lipworth \\ Department of Urologic Surgery, Vanderbilt University Medical Center, Nashville, TN; Division of \\ Epidemiology, Department of Medicine, Vanderbilt University Medical Center, Nashville, TN; \\ International Epidemiology Institute, Rockville, MD; and Vanderbilt O'Brien Center for Kidney \\ Disease, Division of Nephrology, Department of Medicine, Vanderbilt University Medical Center, \\ Nashville, TN
}

\begin{abstract}
OBJECTIVE-To investigate race-sex associations with risk among whites and blacks in the southeastern United States. The relationship between race, sex, and kidney stone risk is poorly understood.
\end{abstract}

\begin{abstract}
METHODS-Participants were 42,136 black and white adults enrolled in the Southern Community Cohort Study between 2002 and 2009, with no history of kidney stones and receiving Medicare or Medicaid services. Incident kidney stone diagnoses through December 2014 were determined via linkage with Centers for Medicare and Medicaid Services research files. Hazard ratios (HRs) for associations with race and sex were computed from multivariable Cox proportional hazards models adjusting for baseline characteristics, comorbid diseases, and dietary intakes.
\end{abstract}

\begin{abstract}
RESULTS-During 116,931 and 270,917 person-years of follow-up for whites and blacks, respectively, age-adjusted incidence rates (95\% confidence interval [CI]) were 5.98 (4.73-7.23) and $4.50(3.86-5.14)$ per 1000 person-years for white men and women, respectively, while corresponding rates among blacks were 2.19 (1.71-2.67) and 2.47 (2.19-2.75) per 1000 personyears. Risk was higher among whites compared to blacks ( $\mathrm{HR}=2.23,95 \% \mathrm{CI} 1.97-2.53)$. Male sex was significantly associated with risk among whites ( $\mathrm{HR}=1.45,95 \% \mathrm{CI} 1.20-1.75)$, but not among blacks (HR $=0.90,95 \% \mathrm{CI} 0.75-1.07)$. Formal tests of interaction by race and sex were statistically significant for all models $(P=.01$ for fully adjusted model).
\end{abstract}

CONCLUSION-The association of incident kidney stones with sex differs between whites and blacks. White men have the highest risk, while no difference in risk is observed between black men and women.

\footnotetext{
Address correspondence to: Ryan S. Hsi, M.D., Department of Urologic Surgery, Vanderbilt University Medical Center, 116121 st Avenue South, A-1302 Medical Center North, Nashville, TN 37232-2765. ryan.hsi@ vanderbilt.edu.

Financial Disclosure: The authors declare that they have no relevant financial interests.
} 
Kidney stone disease prevalence in the United States has risen over the last 3 decades from $5.2 \%$ in $1988-1994$ to $8.8 \%$ in $2007-2010 .{ }^{1}$ This increase has been particularly dramatic among blacks compared to whites. ${ }^{1,2}$ Furthermore, stone disease is more common among white men than white women, ${ }^{1}$ although recent data suggest that the gender gap for stone disease in the United States is narrowing. ${ }^{2,3}$

In contrast, among blacks, stone disease is thought to be more common among women than men. A review of hospitalized stone formers from 1972 to 1981 reported the ratio of black men-to-women was 1:1.55 compared to 2.3:1 for whites. ${ }^{4} \mathrm{~A}$ separate series reported the ratio of black men-to-women undergoing shockwave lithotripsy from 1985 to 1993 was 1:2.1. ${ }^{5}$ Limitations of these studies include retrospective methodology, single-center data, and potential bias from disparities in access to care by sex.

Despite the rising prevalence of stone disease among blacks, the epidemiology is understudied, and it remains unclear how the role of sex differs among whites and blacks in the context of kidney stone risk. To address these questions, we sought to estimate incidence rates of kidney stones and to evaluate race-sex associations with risk in a large prospective cohort of white and black men and women of generally low socioeconomic status in the southeastern United States. We hypothesized that the association between incident stone risk and sex varied by race.

\section{METHODS}

\section{Study Population}

The Southern Community Cohort Study (SCCS) is a prospective cohort study designed to study racial disparities in the risk of cancer and other chronic conditions, including kidney diseases. From 2002 through 2009, approximately 86,000 adult men and women aged 4079, two-thirds black, were enrolled from 12 states in the southeastern United States. The SCCS design and methods have been previously described in detail. ${ }^{6}$ The majority of participants (86\%) were enrolled from community health centers located in underserved communities, while the remaining participants were recruited from mail-based general population sampling. All participants in the SCCS provided written informed consent, and the study was approved by institutional review boards of Vanderbilt University Medical Center and Meharry Medical College.

The current analysis was restricted to white and black SCCS participants who were $\ 65$ years at SCCS enrollment $(n=8480)$, or $<65$ years at enrollment with at least 1 Centers for Medicare and Medicaid Services (CMS) claim after enrollment $(n=42,666)$. These restrictions ensured participants would likely have had continuous coverage in Medicare or Medicaid from the time of SCCS enrollment to the end of the follow-up period (December 31, 2014), for the ascertainment of incident kidney stones. Analyses were restricted to selfreported African American or black and non-Hispanic white participants, as too few persons in other racial groups were available for stable statistical analysis. 


\section{Data Collection}

At enrollment, baseline data on demographic, socioeconomic, anthropometric, lifestyle characteristics, and medical history were ascertained via standardized computer-assisted personal interview at community health centers or via a self-administered mailed questionnaire for the general population participants (questionnaire available at http:// www.southerncommunitystudy.org). Information on weight and height was obtained by selfreport on the baseline questionnaire. Body mass index was calculated as weight in kilograms divided by the square of height in meters. Self-reported medical conditions, including history of diabetes and cardiovascular disease, defined as myocardial infarction, coronary artery bypass surgery, stroke, or transient ischemic attack, were obtained by asking whether a doctor had provided a diagnosis or if there was ever a treatment for the specified condition.

A validated food frequency questionnaire was administered to all SCCS participants to assess dietary intake at baseline. The questionnaire was specifically designed to be race- and sex-specific, and regionally specific to the southern United States. ${ }^{7}$ Macro- and micronutrient intakes were computed by using race- and sex-specific databases of foods consumed in the southern parts of the United States. ${ }^{7-9}$ Detailed methods and the validation of the SCCS food frequency questionnaire with 24-hour dietary recalls have been reported elsewhere. ${ }^{10}$

\section{Assessment of Kidney Stones}

Kidney stone cases were identified by linking the cohort with CMS Research Identifiable Files using Social Security number, date of birth, and first and last name, from January 1, 1999, to December 31, 2014 (the latest date for which linkage data were available). Incident kidney stone events were defined as the first occurrence of a kidney stone-related diagnosis (International Classification of Diseases, Ninth Revision [ICD 9] 592, 592.1, 592.9) within the first 3 diagnosis codes or Current Procedural Terminology codes (50590, 52352, 52353, $52356,50080,50081)$ after SCCS enrollment. Administrative ICD coding for kidney stone diagnoses have been previously shown to have high positive predictive value ( $96 \%)$ compared to manual chart review. ${ }^{11}$ Participants with any stone diagnosis or procedure prior to SCCS enrollment were considered prevalent cases and were excluded $(n=1121)$. Prevalent gallstone disease was ascertained by CMS linkage prior to SCCS enrollment using ICD 9 code 574.

\section{Statistical Analysis}

We excluded participants in the top and bottom $1 \%$ of total caloric intake and those with missing covariates $(n=7889)$. Thus, the final study sample consisted of 1233 incident kidney stone cases (580 whites and 653 blacks) and 40,903 noncases (12,960 whites and 27,943 blacks).

Descriptive statistics for the study population were calculated, including percentages for categorical variables and means (standard deviation) for continuous variables. To address potential confounding of nutrient variables by total energy intake, the nutrient density approach was applied. ${ }^{12}$ Macronutrient intakes (protein, sugar, and fat) were converted to percentages of total energy intake and adjusted for total energy intake using the nutrient 
density method when used in the multivariable models. For micronutrients (sodium, potassium, magnesium, and fiber), reported intakes were divided by total energy intake and multiplied by 1000 to obtain nutrient intake per $1000 \mathrm{kcal}$.

Crude and age-adjusted (United States 2000 standard population) incidence rates of kidney stone events were calculated overall and by race and sex. Univariate associations between baseline demographic, lifestyle, medical, and dietary characteristics with incident stone risk were determined using chi-square tests for categorical variables and $t$ tests for continuous variables. Person-years of follow-up were calculated from date of entry into the SCCS until the date of first stone diagnosis, date of last follow-up in SCCS, death, or December 31, 2014 (the latest date for which CMS data are currently available), whichever occurred first. Mortality was ascertained by linkages with the National Death Index and Social Security Administration vital status service for epidemiologic researchers.

Multivariable Cox proportional hazards models using age as the time scale were used to estimate hazard ratios (HRs) and corresponding 95\% confidence intervals (CIs) for the association between race and sex, with incident stone risk. Adjusted models were constructed a priori in relation to baseline characteristics. The minimally adjusted model included education (<high school, high school or vocational school, some college or higher) and annual household income $(<\$ 15,000, \$ \$ 15,000)$. The second model further added body mass index (categories <18.5, 18.5-24.9, 25-29.9, 30-34.9, 35-39.9, 40 or greater), history of cardiovascular disease, hypertension, diabetes, high cholesterol, and gallstone disease. The fully adjusted model further included quintiles of total energy intake, daily fluid intake, protein, sugar, total fat, calcium (dietary and supplemental), sodium, potassium, magnesium, and vitamin C supplementation more than once per week (yes or no). Vitamin D supplementation in the overall cohort was $<1 \%$ and was not included in the models. Tests for interaction of race and sex were performed by adding the corresponding cross-product terms to the models. The proportional hazards assumption was tested with the Kolmogorov-type supremum test and for interaction with time. Significance was determined based on 2-sided probability with $P$ values $\leq 05$ considered statistically significant. All analyses were conducted using SAS 9.4 (SAS Institute, Inc., Cary, NC).

\section{RESULTS}

Baseline demographic, lifestyle, medical, and dietary characteristics of the SCCS participants included in this study are shown in Table 1. Overall median years of follow-up for whites and blacks were 9.2 and 10.0, respectively. The study population had mean \pm standard deviation age at enrollment of $54.7 \pm 9.3$ years, and was predominantly women $(63 \%)$, black (68\%), and with household income $<\$ 15,000$ per year $(64 \%)$. Sex was not significantly associated with stones overall in univariate analysis $(P=.47)$. Stone formers were more likely to be white compared to nonstone formers. Obesity, cardiovascular disease, diabetes mellitus, high cholesterol, and gallstone disease were more prevalent among stone formers. Mean intakes of total calories, protein, sodium, potassium, and magnesium were higher in the nonstone group, while other macro- and micronutrient intakes were similar between the 2 groups. Specifically among blacks in univariate analysis, sex, obesity, and diabetes mellitus were associated with stone risk compared to black nonstone formers. 
Among whites, sex, insurance coverage type, obesity, diabetes mellitus, and intakes of calcium, potassium, and magnesium were associated with risk compared to white nonstone formers.

During 116,931 and 270,917 person-years of follow-up for whites and blacks, respectively, age-adjusted incidence rates (95\% CI) for kidney stones were 5.01 (4.42-5.60) and 2.36 (2.13-2.59) per 1000 person-years for whites and blacks, respectively (Table 2). When further stratified by sex, among whites, the rates were 5.98 (4.73-7.23) and 4.50 (3.86-5.14) per 1000 person-years for men and women, respectively, while corresponding rates among blacks were 2.19 (1.71-2.67) and $2.47(2.19-2.75)$ per 1000 person-years.

In adjusted analyses, kidney stone risk was higher among whites (HR $=2.23,95 \%$ CI $1.97-$ 2.53) compared to blacks and marginally higher among men compared to women ( $\mathrm{HR}=$ $1.12,95 \%$ CI 0.99-1.28) (Table 3). When blacks and whites were examined separately, male sex was significantly associated with incident stone risk among whites (HR $=1.45,95 \%$ CI $1.20-1.75$ ) but not among blacks ( $\mathrm{HR}=0.90,95 \%$ CI $0.75-1.07)$. Formal tests of interaction by race and sex were statistically significant for all models $(P=.01$ for fully adjusted model), suggesting that the association of incident kidney stone with sex differs between whites and blacks.

Tests for proportional hazard assumption held for sex but not race (0.02). When stratified by follow-up duration, the proportional hazard assumption held among participants with at least 1 year of follow-up. Separate sensitivity analyses were performed restricted to participants with at least 1 year of follow-up, and requiring cases defined as having 2 kidney-stone related ICD codes within the first 3 diagnoses or 1 Current Procedural Terminology code. Both analyses demonstrated no material effect on HR estimates.

\section{DISCUSSION}

In this study of white and black men and women of generally low socioeconomic status, the association of incident kidney stone risk with male sex varies by race. White men had the highest risk for incident stone disease compared to white women and blacks overall, while there was no difference in risk observed between black men and women.

Multiple prior studies in the United States have consistently reported higher prevalent stone risk among whites than blacks in single-institution, ${ }^{4,5,13-15}$ in regional, ${ }^{15,16}$ and in national population-based studies. ${ }^{1} \mathrm{~A}$ few studies have further examined the role of sex and race with kidney stone prevalence among hospitalized patients. Sarmina et al reported a 10-year retrospective review of hospital discharged patients for stone disease and reported that that men:women prevalence of stone disease was 1:1.6 for blacks and 2.3:1 for whites. ${ }^{4}$ Similarly, Michaels et al evaluated 444 patients who underwent shockwave lithotripsy with symptomatic stones, of whom $43 \%$ were white and $27 \%$ were black. ${ }^{5}$ They reported that among blacks, men were less likely to undergo surgery than women (1:2.1). These data more directly reflect treatment patterns for more severe stone disease and may be also biased by access to care and socioeconomic status. In contrast, Scales et al examined self-reported kidney stones in a prospective population-based national survey from 2007 to 2010, and 
prevalent stone disease was higher in white men compared to white women (11.8\% vs $7.5 \%$ ), but more similar between black men and women (4.8\% vs $4.2 \%$, respectively). ${ }^{1}$ Akoudad et al evaluated self-reported prevalent stone disease among 103 black stone formers and 871 white stone formers in the Atherosclerosis Risk in Communities Study (ARIC).${ }^{17}$ Male sex was more strongly associated with risk in whites, while older age, higher triglyceride levels, and gallbladder disease were more strongly associated with stones in blacks (all $P$ interaction $<0.05$ ).

To our knowledge, this is the first study to estimate incidence rates for kidney stones among blacks in the United States. Among black men and women, incidence rates (95\% CI) were 2.19 (1.71-2.67) and 2.47 (2.19-2.75) per 1000 person-years, respectively. Among white SCCS participants, the corresponding rates were 5.98 (4.73-7.23) and 4.50 (3.86-5.14) per 1000 person-years for men and women, respectively, which are higher than what has been previously reported. In the prospective Health Professionals Follow-Up Study of predominantly white men in the United States, incidence rates were highest among age 40$59(3.09-3.77 / 1000 / y)$, were lower among those 60-69 (2.61-2.73/1000/y), and markedly declined after age $70(1.36 / 1000 / y) .{ }^{18}$ Incidence rates among predominantly white nurses in the United States are higher among younger women between age 20 and 40 (1.83/1000/y) and plateau to <1 per 1000 person-years among older women. ${ }^{19,20}$ These differences likely reflect differences in kidney stone risk in the SCCS population compared to other prospective cohorts and differences in methodology. The SCCS cohort was recruited from 12 southeastern states in the United States-termed the "stone belt" due to high burden of kidney stone disease — and predominantly comprised black participants with educational attainment of shigh or vocational school (74\%) and household income $<\$ 15,000$ per year (66\%). Overweight and obesity, established risk factors for stone disease, ${ }^{20}$ are highly prevalent in this SCCS population among both blacks (28\% and $49 \%$, respectively) and whites (30\% and $45 \%$, respectively).

Several studies have examined race-specific 24-hour urinary parameters and the influence of diet on urinary parameters that may, in part, explain the differences in stone risk among whites and blacks. Compared to whites, blacks may have less susceptibility to stone risk due to higher levels of protein-based stone inhibitors, ${ }^{21}$ lower mean urinary calcium, and prevalence of hypercalciuria. ${ }^{22-28}$ Lewandowski et al compared South African whites and blacks on a standardized high oxalate, low calcium diet and found that the rise in urinary oxalate was significantly higher in whites but not in blacks. ${ }^{29}$ Rodgers and Lewandowski evaluated different diets among white and black South African men, and the renal handling of dietary calcium and oxalate was distinct among the race groups, reflected by differences in urinary oxalate, $\mathrm{pH}$, potassium, and citrate. ${ }^{30}$ On univariate analyses, we observed positive associations between obesity and diabetes mellitus on stone risk in both blacks and whites when evaluated separately. Intakes of calcium, potassium, and magnesium were different among our cohort of white stone formers compared to nonstone formers on unadjusted analyses, but not among blacks. While the etiology of stone formation is multifactorial, our data support additional investigation of dietary, lifestyle, metabolic, and genetic risk factors while considering the particular influences of race and sex. 
The SCCS is a unique cohort to examine stone risk in blacks compared to whites due to the large number of black stone formers and dietary intake data. Furthermore the comparability of socioeconomic status between whites and blacks minimizes the potential confounding by access to medical care for stones for the 2 groups. Nevertheless, the influence of potential disparities in access to health care specifically for stone disease is understudied and deserves additional investigation. Limitations of this study include the inherent limitations of using claim-based data for stone diagnoses and procedures, the self-reported nature of the questionnaire data, and the lack of time-dependent covariates. Our definition used to identify cases may over-estimate incident cases if a given participant had a prior stone event not captured in CMS. To mitigate this problem, sensitivity analysis accounting for a run-in time or prevalence period of 1 year did not meaningfully change the findings of this study. While the restriction to those with CMS claims may limit the generalizability of the findings to younger populations, the age groups $\geq 40$ appeared uniformly represented at SCCS enrollment. The findings of this study may not be generalizable to younger populations, those of generally higher socioeconomic status, and populations outside of the southeastern states. Despite these limitations, our findings support the existing literature on the higher incidence of stone risk among men. In particular, this risk appears to be specific to white men. The discrepancy of association of incident kidney stone risk with sex and race warrants further investigation of the sex disparity among whites and why this is not observed among blacks.

\section{CONCLUSION}

White men have the highest incidence of kidney stones compared to white women and to blacks overall. After adjustment for socioeconomic, lifestyle, and medical factors, the increased stone risk among white men persists, while no difference in risk is observed between black men and women.

\section{Acknowledgments}

Funding Support: The Southern Community Cohort Study (SCCS) is funded by grants R01 CA92447 and U01 CA202979 from the National Cancer Institute (NCI), including American Recovery and Reinvestment Act funding (3R01 CA029447-08S1).

\section{References}

1. Scales CD Jr, Smith AC, Hanley JM, et al. Prevalence of kidney stones in the United States. Eur Urol. 2012;62:160-165. [PubMed: 22498635]

2. Tasian GE, Ross ME, Song L, et al. Annual incidence of nephrolithiasis among children and adults in South Carolina from 1997 to 2012. Clin J Am Soc Nephrol. 2016;11:488-496. [PubMed: 26769765]

3. Scales CD Jr, Curtis LH, Norris RD, et al. Changing gender prevalence of stone disease. J Urol. 2007;177:979-982. [PubMed: 17296391]

4. Sarmina I, Spirnak JP, Resnick MI. Urinary lithiasis in the black population: an epidemiological study and review of the literature. J Urol. 1987;138:14-17. [PubMed: 3599198]

5. Michaels EK, Nakagawa Y, Miura N, et al. Racial variation in gender frequency of calcium urolithiasis. J Urol. 1994;152:2228-2231. [PubMed: 7966716]

6. Signorello LB, Hargreaves MK, Blot WJ. The Southern Community Cohort Study: investigating health disparities. J Health Care Poor Underserved. 2010;21:26-37. 
7. Signorello LB, Munro HM, Buchowski MS, et al. Estimating nutrient intake from a food frequency questionnaire: incorporating the elements of race and geographic region. Am J Epidemiol.

2009;170:104-111. [PubMed: 19451177]

8. Malhotra R, Cavanaugh KL, Blot WJ, et al. Higher protein intake is associated with increased risk for incident end-stage renal disease among blacks with diabetes in the Southern Community Cohort Study. Nutr Metab Cardiovasc Dis. 2016;26:1079-1087. [PubMed: 27562875]

9. Kiage JN, Sampson UK, Lipworth L, et al. Intake of polyunsaturated fat in relation to mortality among statin users and non-users in the Southern Community Cohort Study. Nutr Metab Cardiovasc Dis. 2015;25:1016-1024. [PubMed: 26298428]

10. Buchowski MS, Schlundt DG, Hargreaves MK, et al. Development of a culturally sensitive food frequency questionnaire for use in the Southern Community Cohort Study. Cell Mol Biol (NoisyLe-Grand). 2003;49:1295-1304. [PubMed: 14984001]

11. Semins MJ, Trock BJ, Matlaga BR. Validity of administrative coding in identifying patients with upper urinary tract calculi. J Urol. 2010;184:190-192. [PubMed: 20478584]

12. Willett W Nutritional epidemiology. 2nd ed. New York: Oxford University Press; 1998.

13. Mente A, Honey RJ, McLaughlin JR, et al. Ethnic differences in relative risk of idiopathic calcium nephrolithiasis in North America. J Urol. 2007;178:1992-1997, discussion 1997. [PubMed: 17869305]

14. Rous SN. A review of 171 consecutive patients with urinary lithiasis. J Urol. 1981;126:376-379. [PubMed: 7277603]

15. Cary MK. The racial incidence of urolithiasis. J Urol. 1937;37:651.

16. Schey HM, Corbett WT, Resnick MI. Prevalence rate of renal stone disease in Forsyth County, North Carolina during 1977. J Urol. 1979;122:288-291. [PubMed: 469997]

17. Akoudad S, Szklo M, McAdams MA, et al. Correlates of kidney stone disease differ by race in a multi-ethnic middle-aged population: the ARIC study. Prev Med. 2010;51:416-420. [PubMed: 20801154]

18. Curhan GC, Willett WC, Rimm EB, et al. A prospective study of dietary calcium and other nutrients and the risk of symptomatic kidney stones. N Engl J Med. 1993;328:833-838. [PubMed: 8441427]

19. Curhan GC, Willett WC, Speizer FE, et al. Comparison of dietary calcium with supplemental calcium and other nutrients as factors affecting the risk for kidney stones in women. Ann Intern Med. 1997;126:497-504. [PubMed: 9092314]

20. Taylor EN, Stampfer MJ, Curhan GC. Obesity, weight gain, and the risk of kidney stones. JAMA. 2005;293:455-462. [PubMed: 15671430]

21. Rodgers AL, Mensah PD, Schwager SL, et al. Inhibition of calcium oxalate crystallization by commercial human serum albumin and human urinary albumin isolated from two different race groups: evidence for possible molecular differences. Urol Res. 2006;34:373-380. [PubMed: 17072609]

22. Taylor EN, Curhan GC. Differences in 24-hour urine composition between black and white women. J Am Soc Nephrol. 2007;18:654-659. [PubMed: 17215441]

23. Meier DE, Luckey MM, Wallenstein S, et al. Calcium, vitamin D, and parathyroid hormone status in young white and black women: association with racial differences in bone mass. J Clin Endocrinol Metab. 1991;72:703-710. [PubMed: 1997523]

24. Blackwood AM, Sagnella GA, Cook DG, et al. Urinary calcium excretion, sodium intake and blood pressure in a multi-ethnic population: results of the Wandsworth Heart and Stroke Study. J Hum Hypertens. 2001;15:229-237. [PubMed: 11319670]

25. Maloney ME, Springhart WP, Ekeruo WO, et al. Ethnic back-ground has minimal impact on the etiology of nephrolithiasis. J Urol. 2005;173:2001-2004. [PubMed: 15879804]

26. Charlton KE, Steyn K, Levitt NS, et al. Ethnic differences in intake and excretion of sodium, potassium, calcium and magnesium in South Africans. Eur J Cardiovasc Prev Rehabil. 2005;12:355-362. [PubMed: 16079643]

27. Whalley NA, Moraes MF, Shar TG, et al. Lithogenic risk factors in the urine of black and white subjects. Br J Urol. 1998;82:785-790. [PubMed: 9883212] 
28. Whalley NA, Martins MC, Van Dyk RC, et al. Lithogenic risk factors in normal black volunteers, and black and white recurrent stone formers. BJU Int. 1999;84:243-248. [PubMed: 10468714]

29. Lewandowski S, Rodgers A, Schloss I. The influence of a high-oxalate/low-calcium diet on calcium oxalate renal stone risk factors in non-stone-forming black and white South African subjects. BJU Int. 2001;87:307-311. [PubMed: 11251520]

30. Rodgers AL, Lewandowski S. Effects of 5 different diets on urinary risk factors for calcium oxalate kidney stone formation: evidence of different renal handling mechanisms in different race groups.

J Urol. 2002;168:931-936. [PubMed: 12187193] 


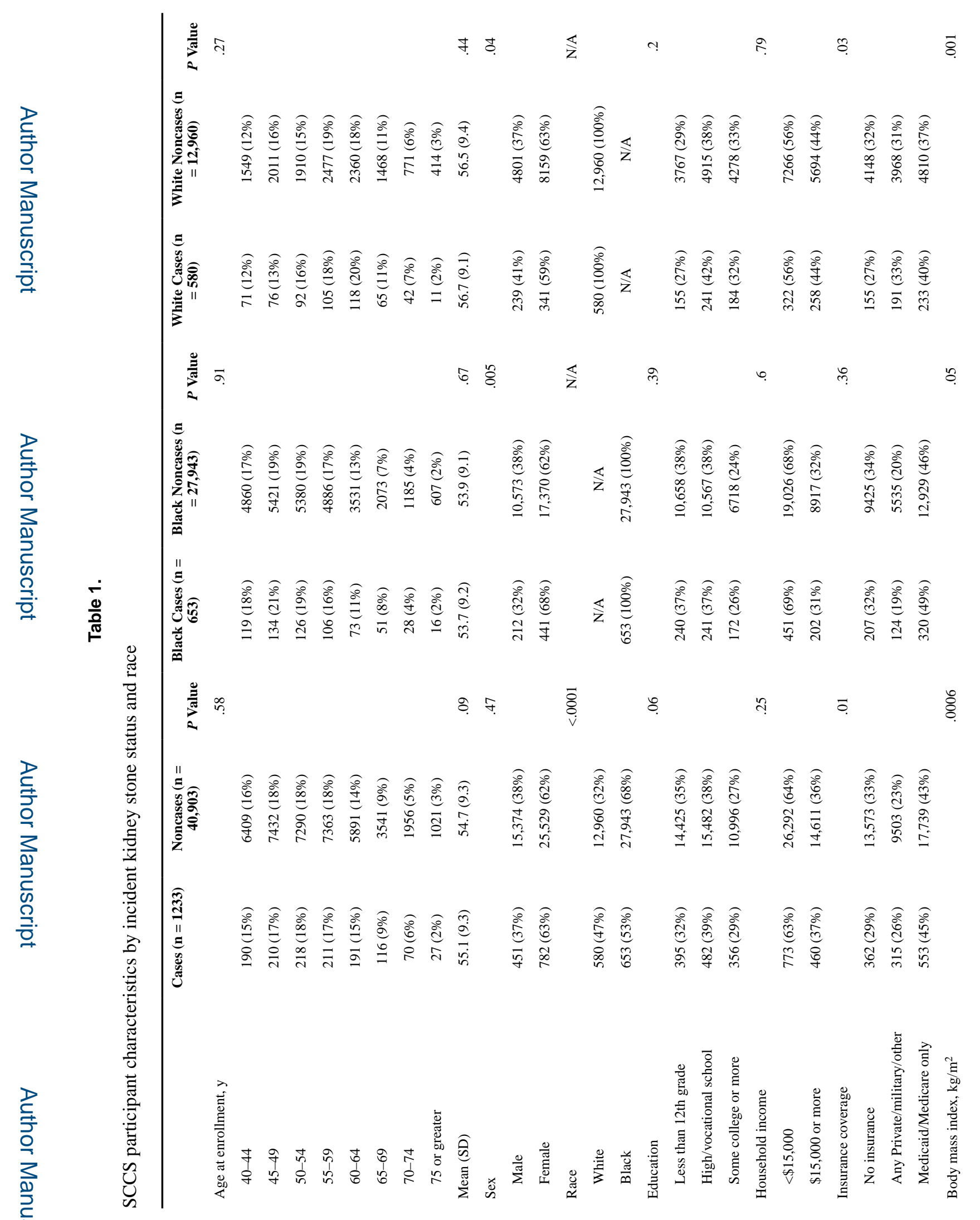




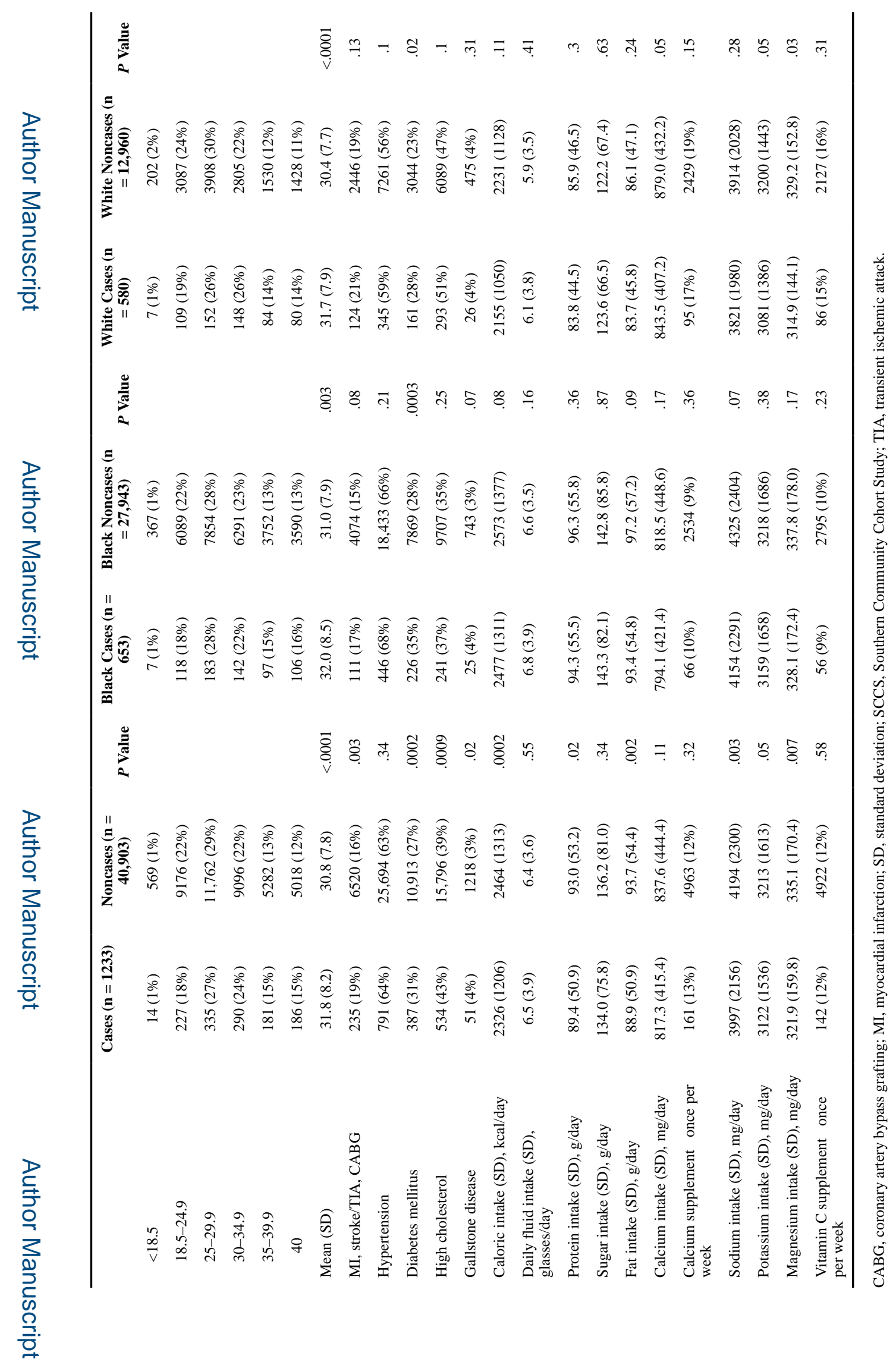

Urology. Author manuscript; available in PMC 2020 March 02. 


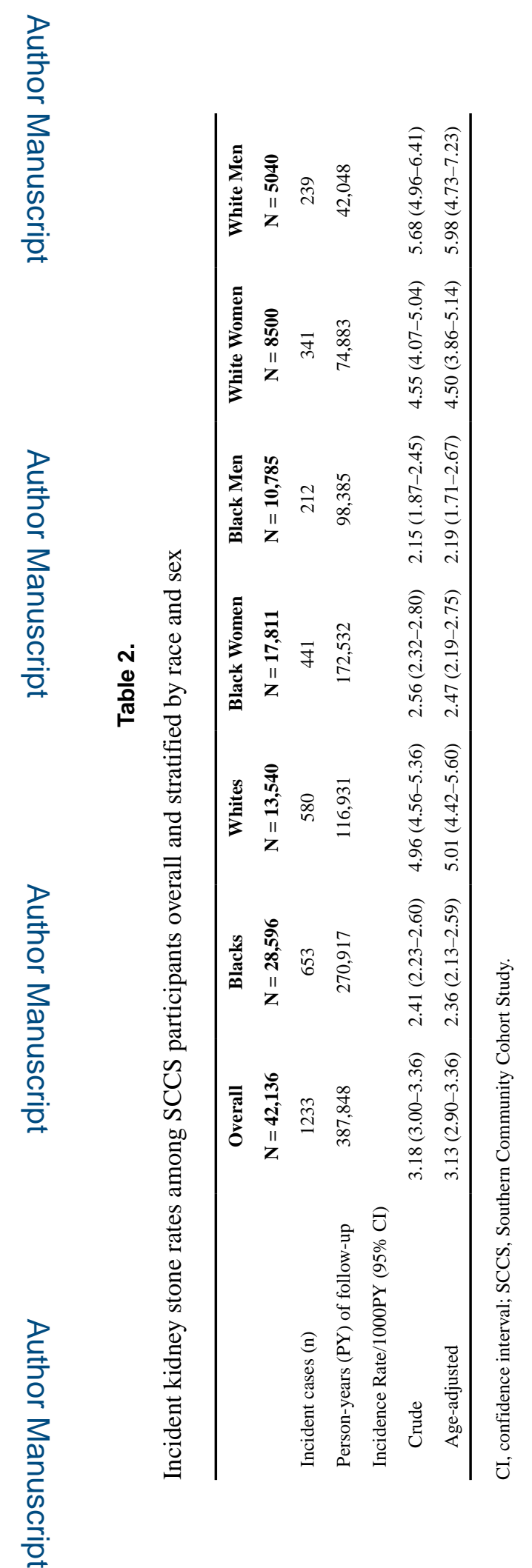

Urology. Author manuscript; available in PMC 2020 March 02. 

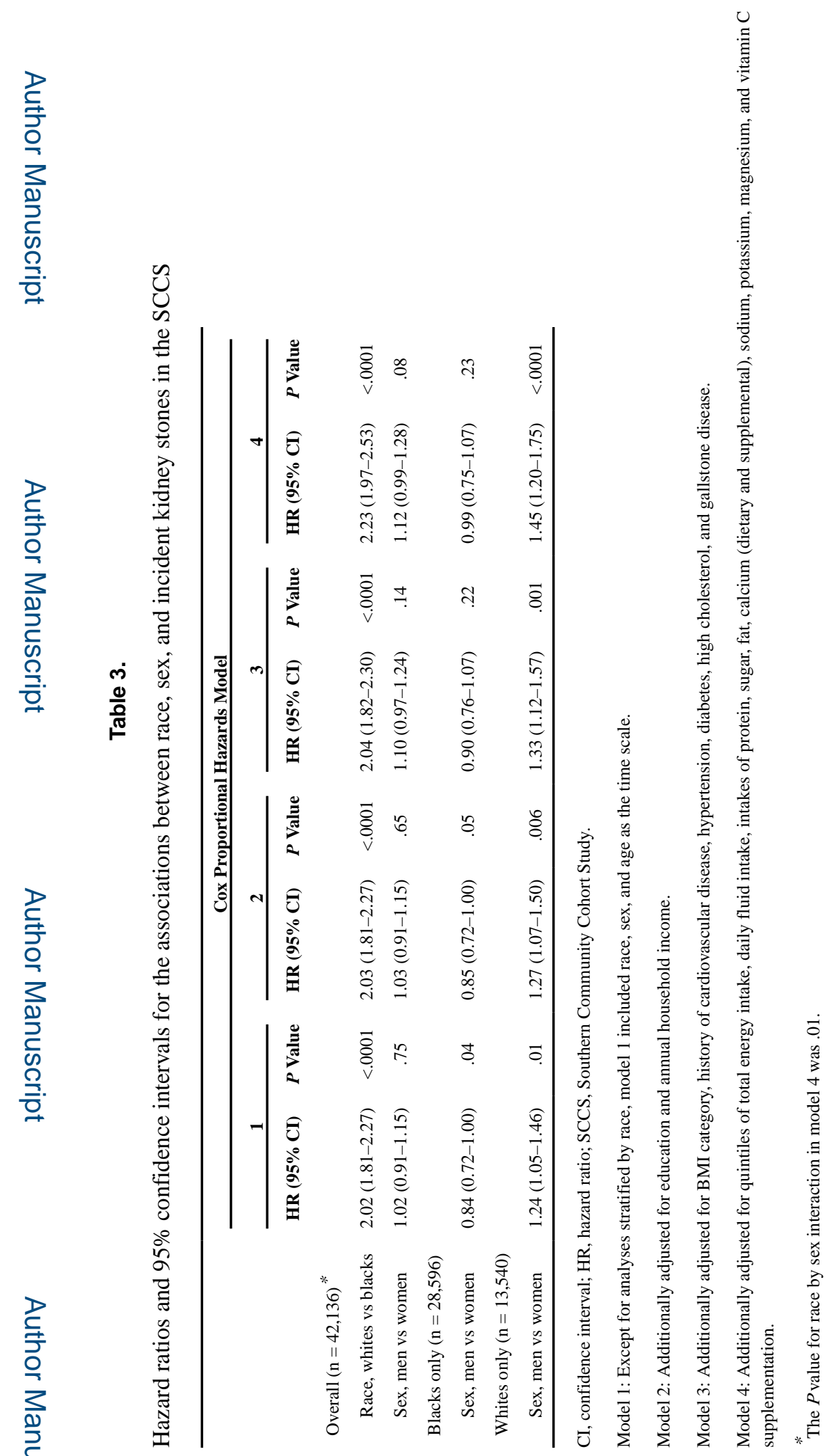

Urology. Author manuscript; available in PMC 2020 March 02. 